\title{
L'HOMME L'Homme
}

Revue française d'anthropologie

153 | janvier-mars 2000

Observer Nommer Classer

\section{Parler parenté}

\section{Claude Meillassoux}

\section{OpenEdition}

\section{Journals}

Édition électronique

URL : http://journals.openedition.org/lhomme/9

DOl : $10.4000 /$ /homme.9

ISSN : 1953-8103

\section{Éditeur}

Éditions de l'EHESS

\section{Édition imprimée}

Date de publication : 1 janvier 2000

Pagination : 153-164

ISBN : 2-7132-1316-9

ISSN : 0439-4216

Référence électronique

Claude Meillassoux, «Parler parenté », L'Homme [En ligne], 153 | janvier-mars 2000, mis en ligne le 04 mai 2007, consulté le 19 avril 2019. URL : http://journals.openedition.org//homme/9 ; DOI : 10.4000/ Ihomme.9 


\title{
Parler parenté
}

\author{
Claude Meillassoux
}

Les RECHERCHES sémantiques d'Émile Benveniste $(1966,1969)$ ont été peu sollicitées par les anthropologues, bien qu'elles constituent une démarche cognitive qui donne matière à découvrir la cohérence des systèmes de parenté, notamment européens.

Dans le matériel de terrain, l'approche généalogique de la parenté introduit une ambiguiité fondamentale entre le biologique et le social. La présomption génétique qui préside à la collecte généalogique réintroduit en permanence le principe de consanguinité biologique et entretient l'illusion qu'il en est le substrat universel ${ }^{1}$. Or, même lorsqu'il existe une coïncidence entre parenté sociale et connexions biologiques, c'est toujours de la socialisation de celles-ci qu'il est question dans le discours de l'informateur. Les tentatives faites pour y retrouver le substrat biologique, si elles n'ont pas une intention comparative expresse et déclarée, sont de pure école.

Certes, il est toujours possible, a posteriori, de convertir en termes de parenté sociale les matériaux recueillis à travers la grille consanguine, mais ils ne véhiculent plus alors leurs données propres, celles-ci étant rejetées par l'explication implicite qu'en donne la consanguinité. Le détournement de la perception du phénomène de parenté vers un vocabulaire approximatif contribue à l'effacement de principes institutionnels tout aussi pertinents tels que, par exemple, la résidence ou l'itinérance, et même à la réinvention par les anthropologues ou, parfois, à l'adoption par les popu-

1. Sans parler des références que nombre d'auteurs font au rapport dit " de sang " là même où cette symbolique n'existe pas.

Communication présentée à la III e Conférence de l'AEAS, Oslo 24-27 juin 1999, atelier "A Fresh Agenda for Kinship Studies ». 
lations concernées de nouvelles «traditions » plus conformes aux conceptions ethnologiques occidentales ou aux règles administratives ${ }^{2}$.

La procédure de collecte généalogique proposée par le célèbre manuel Notes and Queries on Anthropology, édité par le Royal Anthropological Institute - qui devint la bible des anthropologues et qui, de 1874 à 1964, connut de nombreuses rééditions -, se conforme en tous points à la méthode préconisée par Morgan ${ }^{3}$, laquelle se fonde, elle aussi, sur le préjugé consanguin ${ }^{4}$. La «mère» est celle "qui a porté », le père celui "qui a engendré » et il convient, dans tous les cas, de s'assurer de la réalité de ces faits (Notes and Queries, 1964: 54-55). La procédure recommandée semble prévenir toute erreur : " D'abord, il faut demander à l'informateur le nom de sa mère, la femme du ventre de laquelle il est né, puis il faut lui demander le nom de l'homme qu'elle a épousé, celui qui l'a engendré. Ensuite, comment il s'adresse à chacun d'eux ou quel est le nom indigène pour désigner cette relation. Enfin, l'enquête doit porter sur la façon dont chaque parent s'adresse à l'informateur ". L'ethnologue aurait alors accès aux termes signifiant " mère ", "père " et " enfant ". On recueille les termes désignant les collatéraux en demandant si la "mère » et le "père " (qu'on appelle par leur nom respectif) ont eu d'autres enfants et par quels termes on les nomme lorsqu'on s'adresse à eux: "Ainsi obtient-on le registre généalogique d'une famille élémentaire et les termes pour père, mère, enfant, germains. Utilisant les termes vernaculaires dont il s'est alors assuré du sens, l'enquêteur est en mesure de poursuivre l'enquête généalogique et d'enregistrer les autres personnes liées par la consanguinité et l'affinité, et les termes d'adresse."

Comme y furent donc invités de nombreux ethnologues et leurs élèves (dont moi-même), dès que l'étude généalogique leur permettait d'établir les connexions biologiques, ils s'autorisaient à les qualifier par les termes de parenté propres à nos cultures. En fait, les termes vernaculaires recher-

2. En Australie, les Aborigènes, pour recouvrer leurs droits sur leurs anciens territoires, doivent prouver leur "traditionalisme" et font appel à des ethnologues professionnels pour leur construire des systèmes de parenté conventionnellement acceptables par l'administration.

3. "A brief reference to our own system of consanguinity will bring into notice the principles which underlie all systems" (Morgan [1877] 1963: 404).

4. David Schneider (1984) fait remarquer que Rivers (1924: 75), bien quayant mis en garde les chercheurs contre la conception biologique de la parenté, insistait pour que ses informateurs emploient les équivalents de "père ", "mère ", etc., au sens génétique anglais. "I was able to make the natives understand very thoroughly that I wanted the "proper father" ", écrit-il. On conçoit le quiproquo qui peut naître de cette exigence, si, pour l'informateur, son "proper father » est celui qui lui a été désigné comme tel et qui peut être à son insu un père social, puisqu'il est, moins que quiconque, capable de témoigner de celui qui a fécondé sa génétrice, et qu'il est tout autant incapable d'identifier celle-ci, sinon par ouïdire. Connaître à coup sûr les géniteurs d'un individu implique une enquête auprès de tierces personnes qui auraient été témoins personnellement de la conception, de la "fidélité " de l'épouse, de l'accouchement, de la non-substitution du nouveau-né jusqu'au moment de la dévolution des noms, etc. Le "père " désigné est toujours un père social et donc "the proper father». 
chés par l'observateur dans Notes and Queries sont "génitrices", "géniteurs", et autres individus liés biologiquement, mais non "mère", "père ", etc., qui supposent un statut social reconnu que n'accorde pas a priori la connexion biologique.

Il est indispensable de rappeler ici les caractéristiques spécifiques de ces termes pour mesurer leur congruence par rapport à ceux auxquels nous confronte le terrain. Le vocabulaire parental, que j'appelle latin, dans lequel puise l'ethnologie classique, renvoie à deux catégories de relations de parenté, dites de consanguinité et d'affinité.

Pour la première catégorie, dont je traiterai ici, six termes élémentaires désignent des relations parentales réputées sans équivoque (parce que supposées biologiquement contiguës), donc applicables universellement: " père ", " mère ", "fils ", " fille ", frère ", « sœur ». Tous les autres rapports de parenté sont considérés comme dérivant de ces six rapports et décrits par une combinaison des termes précédents : "sœur du père ", "père du père ", "fille du frère du père ", etc., procédé qui permet de prolonger sans limites les généalogies et le champ d'une parentèle ${ }^{5}$ en précisant de surcroît, si elle est en ligne paternelle ou maternelle.

D'autres termes, apparemment élémentaires car s'exprimant par un seul vocable (" oncle ", " tante ", " cousin ", " cousine ", " nièce » et "neveu »), sont écartés par certains comme ambivalents, car ils n'indiquent pas l'appartenance paternelle ou maternelle. Ils doivent en effet s'accompagner d'un qualificatif ( "utérin » ou " germain, " paternel» ou " maternel») pour être significatifs. L'accord sur le choix des six termes élémentaires semble général. Il remonte à Morgan ([1877] 1963) et prévaut autant dans les analyses fonctionnalistes que structuralistes. Rares sont pourtant les définitions de ces termes qui s'accordent avec celles données par leurs utilisateurs et l'on doit supposer qu'ils sont pris dans l'acception générale que leur accorde notre culture. Voici celles que, par exemple, donne le dictionnaire Le Robert (1972) :

"Père: Homme qui a engendré, qui a donné naissance à un ou plusieurs enfants. En droit : ascendant mâle au premier degré... (pedre au XI ${ }^{\mathrm{e}} \mathrm{s}$.).

Mère: Femme qui a donné la vie à un ou plusieurs enfants... (medre au XI' s.). [Pas de définition en droit.]

Fils : Être humain du sexe masculin, considéré par rapport à son père et à sa mère ou l'un des deux seulement... ( $\mathrm{X}^{\mathrm{e}} \mathrm{s}$.).

Fille : Personne du sexe féminin, considérée par rapport à son père et à sa mère ou l'un des deux seulement... ( $\mathrm{XI}^{\mathrm{e}} \mathrm{s}$.).

5. Parentèle : ensemble des individus apparentés cognatiquement à ego et répondant aux diverses obligations qu'elle entraîne. 
Frère: Personne du sexe masculin, considérée par rapport aux enfants des mêmes parents... (1100; fradre au IX $\mathrm{IX}^{\mathrm{e}}$.).

Scur: Personne du sexe féminin ( $\mathrm{XI}^{\mathrm{e}} \mathrm{s}$.), considérée par rapport aux enfants des mêmes parents. $»^{6}$

Le Trésor de la langue française adopte la même définition pour "père " : «a) Homme qui a engendré, qui a un ou plusieurs enfants...; b) Dans le langage courant : - Le mâle (par rapport à l'animal qui a engendré). » Pour " mère ", dont l'ascendance biologique est pourtant plus évidente, la fonction sociale est située sur un même plan : "Femme qui a mis au monde, élève ou a élevé un ou plusieurs enfants. " Tous ces termes se déduisent de la définition du père et de la mère, ayant "engendré " ou "donné la vie » à des enfants. Ils reposent donc sur un substrat génésique. Ils sont porteurs d'une théorie, celle de la consanguinité, et leur emploi n'est pas neutre. Ils ne sont définis ici par aucune autre des fonctions parentales : nourricière, éducative, protectrice, de soutien, etc. Notons cependant que le père est considéré «en droit».

En ce qui concerne les termes anglo-saxons, selon le Webster's Third New International Dictionary (1981), la référence biologique est également centrale :

"Father: A man who has begotten a child (p. 828b).

Mother: A woman who has given birth to a child (p. 1474b).

Brother: A male human being considered in his relation to another person having the same parents or one parent in common (p. 284a).

Sister : A female human being related to another person having the same parents ${ }^{7}$. Son: The male offsprings of human beings (p. 2172b).

Daughter: A human female having the relation of child to a parent (577b). ${ }^{8}$ À la différence du français, la langue anglaise possède un terme, daughter (grec: thugater), qui se distingue de girl («fille»), pour désigner le féminin du mot français « fils »?.

En France, les six termes élémentaires retenus en ethnologie de la parenté semblent apparaitre vers le X ${ }^{\mathrm{e}}$-XII ${ }^{\mathrm{e}}$ siècle (Le Robert, 1972), dans un contexte de mise en place aristocratique des rapports familiaux. Selon

6. La datation de ces termes en montre le caractère relatif.

7. Ces deux dernières définitions renvoient à celle de parent (Webster's TNID: 1641a) qui lui donne un double sens, biologique et nourricier; parent: " one that begets or brings forth offsprings " (mes italiques). 8. La daughter est une child et non un offspring comme le son. Child: "an unborn or recently born human being" (Webster's TNID : 388a). Offspring: "something that springs from an animal or plant reproducing its kind" (ibid.: 1568a).

9. Le français n'a pas non plus un équivalent du wife anglais, converse de «mari» et distinct de spouse ("épouse ", " époux "), bien que le vieux français ait connu ossur, du latin uxor. Le français ne connaît pas non plus d'équivalent de sibling qui désigne soit les descendants de la ligne paternelle ou maternelle à l'exclusion de l'autre, soit les frères et sœurs. En revanche l'anglais n'a pas l'équivalent d'un terme spécifique comme "aïeul ". 
Bertrand Russell, en Grande-Bretagne et au Pays de Galles, la succession père/fils, par exemple, n'est repérée qu'à partir de 1250. Ces mots ont donc acquis, dans nos sociétés, un sens historique qui ne se rencontre pas nécessairement dans les sociétés exotiques.

Dans les deux langues, française et anglaise, les termes de parenté élémentaires possèdent des propriétés descriptives et associatives spécifiques. Au niveau de la morphologie verbale, suivant Murdock (1949: 98) qui renvoie à Lowie, on distingue les termes élémentaires des termes composés. Sont élémentaires ceux qui ne sont formés que d'un seul vocable, comme les six énumérés ci-dessus. Ils décrivent des rapports primaires qui semblent aller de soi en raison de la contiguïté génésique des parents nommés. Les termes composés sont de deux sortes: descriptifs lorsqu'ils comprennent plusieurs termes élémentaires, tel "frère de la mère ", ou dérivés, lorsque ce sont des termes élémentaires altérés comme, par exemple, "grand-père ", "petit-fils".

Dans les systèmes de parenté concernés, les six termes élémentaires n'ont de sens que par couple: ce sont des termes que j'appellerai converses. "Mère » ou "père » renvoient à « fils/fille»; «frère » ou « sœur » renvoient l'un à l'autre et à eux-mêmes (ils sont converses dans le premier cas et également réciproques dans le second), etc. ${ }^{10}$ Outre le rapport de parenté immédiat, les termes élémentaires indiquent, que ce soit en français ou en anglais, le genre de la personne et sa "génération ».

Depuis McLennan (1865) l'ethnologie fait, à juste titre, la différence entre termes de référence et termes d'adresse (dits aussi vocatifs) ${ }^{11}$. Les termes de référence désignent un statut parental, quel que soit le locuteur. Ainsi dit-on "mon père" ou le "père" de X. Le terme d'adresse est celui qu'emploie un individu pour interpeller familièrement un de ses parents. Il peut être le même que le terme de référence ("père ") ou différent («papa $)^{12}$. En nous limitant aux termes de référence, on peut encore dis-

10. Certains auteurs emploient " réciproque " pour signifier ce que j’appelle « converse ». " Frère », terme réciproque en français, traduit souvent un terme converse dans d'autres langues. Nous ne nous arrêterons pas ici, malgré leur intérêt, à l'emploi de termes converses par métonymie (comme "père " ou "mère " employés comme termes d'adresse entre époux, par exemple).

11. D'aucuns emploient le mot "descriptif» dans le sens de "vocatif».

12. Les termes élémentaires référentiels de parenté peuvent aussi être utilisés comme termes d'adresse indépendamment de toute relation parentale, consanguine ou sociale, par exemple, dans les rapports de voisinage, dans les ordres religieux, entre individus appartenant à une même classe d'âge, à un même ordre ou un même parti ou pour marquer une profonde amitié. Ces usages soulèvent le problème de savoir si ces termes de parenté sont employés en extension ou en réduction; si le sens restreint s'est étendu à des catégories plus larges ou si, au contraire, le sens large s'est appliqué à des catégories plus restreintes; par exemple, le terme désignant le chasseur adulte, pourvoyeur de nourriture dans les sociétés cynégétiques, a pu désigner, par réduction successive, une catégorie d'âge, celle des adultes mâles pourvoyeurs de nourriture, puis dans les sociétés domestiques ou dynastiques, une catégorie parentale restrictive (le père). 
tinguer les termes que j'appelle univoques ${ }^{13}$ des termes dits classificatoires. Dans notre culture monogame, "père" et "mère" sont univoques: chaque individu (Ego) ne se rapporte qu'à un seul père, une seule mère. Tous les autres termes de parenté sont classificatoires: Ego peut se rapporter à plusieurs fils, filles, sœurs, frères, oncles, tantes, neveux, nièces, etc. - autant de catégories parentales qui entrent respectivement dans une même classe par rapport à lui.

Dans notre système de parenté, les termes, qu’ils soient univoques ou classificatoires, se distinguent aussi selon que le rapport parental se conçoit comme direct ou récursif (Hirschfeld 1986). Le rapport parental est récursif lorsque la parenté entre deux individus se conçoit, explicitement ou implicitement, par référence à un autre parent d'une génération antérieure ou postérieure : les rapports entre "frères " ou entre " oncle » et «neveu ", par exemple, sont conçus par référence au père ou à la mère. Le rapport parental est direct lorsque cette référence à un tiers parent est absente, comme c'est le cas entre le père ou la mère et leurs enfants.

\section{Incongruités sémantiques}

Dans la pratique ethnologique courante, les relations parentales observées dans la société étudiée sont décrites ou traduites par le recours à un ou plusieurs termes latins (ou la combinaison entre eux). Mais deux obstacles interviennent et rendent cette opération très approximative. D'une part, comme dans tous les cas de description ou de traduction, les champs sémantiques des termes confrontés ne sont pas identiques. Ils peuvent ne se recouvrir que partiellement. D’autre part, ces termes sont polysémiques et même si les champs sémantiques coïncident, la traduction ne peut s'attacher qu'à un seul sens. Lorsque cette opération de traduction s'assortit d'une analyse scientifique, le choix du champ sémantique dans la langue de traduction équivaut à la mise en ouvre de la théorie inhérente à la langue choisie. Les définitions du dictionnaire, français ou anglais, contiennent la théorie de la consanguinité à l'état latent. Il existe ainsi, à ma connaissance, de nombreuses populations parmi lesquelles la notion converse de «fils" et de "fille» (daughter) n'apparaît pas. Dans la plupart des civilisations adelphiques (et de classes) sahélo-soudaniennes que je connais (soninké, maninka, bamana, etc.), la progéniture est désignée d'un terme générique, par exemple den (den-tye ou den-muso selon le sexe,

13. Parfois dits aussi descriptifs. Le mot anglais denotative a un sens différent puisque, selon Murdock (1949 : 98), il englobe "souvent plusieurs personnes de connexion parentale identique ", comme frères, fils, etc., que je considère comme des catégories classificatoires. Benveniste (1969, I) oppose « classificatoire " et " descriptif ". 
en bamana) qui signifie «bourgeon", « rejet» (rejeton), ou "plus petit» par rapport à un "plus grand", etc. Néanmoins, den est toujours traduit, dans les ouvrages français, par un terme de consanguinité : "fils" ou "fille», au lieu d'" enfant ». Or, dans la pratique parentale, le mot vernaculaire n'implique de relation ni converse ni univoque avec les parents que nous appelons "père " ou " mère ». En revanche, le mot den s'accompagne d'une relation classificatoire qui n'existe pas chez nous, puisque l'enfant considère plusieurs personnes comme ce que nous appelons ses "pères " ou "mères ${ }^{14}$. Ainsi ce vocabulaire adelphique, qui nous paraît pauvre ou incongru dans notre perspective génésique, exprime-t-il une situation inexistante institutionnellement dans notre système de parenté. Contrairement à l'hypothèse de Morgan, qui percevait dans le système classificatoire les séquelles d'une promiscuité sexuelle (donc consanguine) " primitive ", il faut y voir plutôt la substitution toujours latente, par rapport à chaque enfant, de tous les personnages qu'il place dans une même classe. Tous les hommes qu'il considère comme ses "pères" sont susceptibles de remplir cette fonction envers lui en toutes circonstances.

Dans notre langage, "frère» ou «sœur» expriment la collatéralité simple et réciproque. Mais il est fréquent que, dans les unités domestiques, la collatéralité ne s'exprime qu'en association avec l'aînesse: on nomme par des termes spécifiques des aînés ou des cadets dans la génération (définie socialement) ou dans la classe d'âge. Le vocable "frère", au sens que nous lui donnons, n’a plus d'équivalent. Des termes élémentaires distincts désignent respectivement le collatéral aîné et le collatéral cadet (par exemple, gida et xoxoone en soninké, que nous traduisons par les locutions "frère aîné" ou "frère cadet») sans avoir de racine commune. Ainsi des termes que nous traduisons par "frère » ou «sœur " - qui chez nous sont réciproques - expriment en fait un rapport à la fois converse et d'aînesse, totalement absent de notre conception. Il existe aussi des termes vernaculaires, identiques pour les deux sexes, qui désignent le collatéral aîné ou cadet, tantôt du même genre qu'Ego, tantôt de l'autre genre. En désignant en même temps l'aînesse relative, le genre et la collatéralité, des termes vernaculaires expriment ainsi une notion parentale dont n'est capable aucun des nôtres, même composés.

14. Émile Benveniste (1969, I : 235 sq.) constate "cette instabilité du nom du "fils" ", dont l'étymologie renvoie au nourrisson ou au rejeton. Il oppose cette indétermination à la stabilité de nepos "neveu ". Tous les enfants d'une même génération peuvent en effet être classés sans équivoque dans cette catégorie par rapport aux adultes de la génération précédente, expressément parce qu'aucun lien ne les lie de façon privilégiée à l'un d'entre eux; "fils » suppose une filiation univoque qui n'opère pas dans la société domestique. Ainsi, par rapport à notre vocabulaire, les termes « père » et " oncle " paternel se confondent, de même que "fils" et "neveu ". 
À cette absence de congruence de notre vocabulaire s'ajoute la carence de certains de nos termes. On traduit souvent par "grand-père » ou "père du père ", qui sont chez nous des termes dérivés ou descriptifs, ce qui semble correspondre dans d'autres langues à un terme élémentaire, devenu désuet en français, tel "aïeul(e)». Or, dans le vocabulaire français, "aïeul» était le converse d'un autre terme élémentaire de parenté, "avelet " et "avelette", qu'on ne connaît plus (Benveniste 1969, chap. 3). La désuétude de ces termes révèle qu'un rapport parental direct entre l'aïeul et ses avelets a disparu pour être remplacé par un double rapport récursif, celui du fils du fils au père du père ${ }^{15}$. La locution "grand-père" témoignerait donc d'une évolution de notre système de parenté vers une polarisation paternelle qui brise le rapport immédiat entre l'aïeul et l'avelet et les renvoie de part et d'autre du père, positionné au centre du dispositif parental. Or cette évolution de la parenté dans les groupes aristocratiques n'a pas eu lieu dans toutes les sociétés. L'usage indiscriminé des termes "grand-père » et "petit-fils » attribue pourtant ipso facto cette transformation historique à toutes les sociétés où on les introduit, donc aussi les caractéristiques sociales aristocratiques qui les accompagnent. C'est en effet dans la parenté de type dynastique ${ }^{16}$, là où il y a polarisation de la famille autour du père et nouement d'un lien privilégié avec le fils aîné, que la notion de "père " cesse d'être classificatoire. Ce modèle, qui nous semble naturel, est lui aussi historique. Les frères germains du père ne sont plus des "pères " pour les enfants de celui-ci, mais des " oncles", tandis que les enfants communs de l'adelphie ${ }^{17}$ deviennent leurs « neveux » et « nièces ", lesquels, de « sœurs » et « frères » qu'ils étaient entre eux, deviennent « cousins/cousines ». Les notions d'oncle, de tante, cousin, cousine, nièce et neveu n'existent, dans la société domestique patrilinéaire, que pour désigner la relation avec les parents maternels. Mais elles n'ont pas leurs équivalents paternels. Avec le système dynastique, ces notions s'introduisent entre les parents paternels, ce qui implique une indiscutable distanciation.

La congruence relative des rapports de parenté d'un système social exotique avec le nôtre a fait l'objet de recherches grâce notamment à l'analyse componentielle (ou sa variante transformationnelle) ${ }^{18}$. Il s'agit de tentatives pour établir un passage plus précis entre les vocabulaires, vernaculaire et latin. Selon cette procédure, on classe sous chaque terme vernaculaire relatif à la parenté toutes les relations de parenté auxquelles il correspon-

15. Benveniste rappelle à ce propos des glissements de sens indicatifs du passage d'un mode parental historique à un autre (selon moi, passage du système adelphique au système dynastique).

16. Ce système parental s'élabore et évolue dans les sociétés aristocratiques.

17. Système parental de la société domestique, à succession collatérale, de senior à junior.

18. Voir Tax 1937; Goodenough 1956; Lounsbury 1956, 1964 ; Wallace \& Atkins 1960 ; Notes and Queries 1964: 81-82 ; Coult 1967; Panoff 1965, 1976 ; Augé, ed. 1975: 48, etc. 
drait dans notre système et qui seraient exprimées dans notre langue. Il s'agirait ainsi de faire apparaître le champ sémantique des termes concernés. La démarche permet, certes, de dégrossir le problème et elle peut faire apparaître certains aspects relatifs à la conceptualisation comparée des sexes et des âges, par exemple. Mais cette analyse ignore le caractère polysémique des termes vernaculaires en les réduisant au sens consanguin de nos termes "latins». Les fonctions d'engendrement, nourricières, éducatives, protectrices, etc., se confondent avec les notions de "père" ou "mère" sans que l'analyse componentielle ne soit en mesure de pouvoir repérer lesquelles sont en cause. Ainsi, «mère " contient deux types d'informations : le sexe du sujet et sa relation institutionnelle avec un autre individu. L'une, qui renvoie à l'appartenance au genre féminin, est objective, semblable dans les systèmes exotique et latin (encore que conventionnelle dans certains cas) ; elle peut être traitée comme une donnée comparable et faire apparaître des combinaisons ou des classifications explicites et distinctives. Mais il n'en est pas de même de l'autre, c'est-à-dire du statut économique et social de la "mère" qui résulte de conventions sociales distinctives. L'analyse ne vaut que pour le signifié des termes de parenté qui se réfere à des faits bruts, mais elle cesse d'être "objective" dès qu'elle porte sur le contenu institutionnel, relatif et arbitraire, des rapports sociaux spécifiques à chacun des systèmes comparés ${ }^{19}$. En outre, si on compare une société polygame à une société monogame, la notion de «mère » est classificatoire dans la première, directe dans la seconde : le critère de consanguinité est absent dans le premier cas, lorsque les enfants d'une même mère n'ont pas tous le même père, mais pas dans l'autre cas.

Aussi, à ce niveau sémantique conçu comme "élémentaire ", donc fondamental, aucun des six termes "fils", "fille» (daughter), "père» ou "mère ", "frère » ou "sœur" n'ont d'équivalents stricts. Les distinctions vernaculaires entre classificatoire ou univoque, converse ou non, élémentaire ou composé, réciproque ou non, ne sont pas respectées par l'emploi de nos termes. Des notions inconnues sont introduites par leur truchement dans les systèmes étudiés, comme celle de paternité unilinéaire (rapport exclusif père/fils) ou de collatéralité non hiérarchique ("frère" sans distinction d'âge), tandis que d'autres sont ignorées, comme l'aînesse relative, l'aviolité, la non-sexualisation de la collatéralité, etc. Si ces termes sont impropres lorsqu'ils sont limités à leur signification simple, on conçoit le degré d'indétermination auquel mène leur utilisation en chaîne par le pro-

19. Un essai d'analyse componentielle de Nelson Graburn (1964: 203), constatait, en s'appuyant sur la remarque de Spencer (1914) concernant la résidence, "that an analysis whose components are derived from behavioral norms provides criteria for differentiating kin classes that are likely to be more meaningful to the actors within the system that those criteria which are purely genealogical ». 
cédé dit « descriptif». Qui est le « le fils du frère du père » dans un système classificatoire? Est-il l'un des enfants mâles du collatéral aîné ou le cadet d'un des membres d'une adelphie? Peut-on parler au singulier, comme c'est la coutume dans notre discipline, quand il s'agit de terme désignant des classes de parents? (par exemple "La diagonale de la belle-mère " (?); Glowczewski \& Pradelles de Latour 1987). Au regard de la thèse "combinatoire" (Héritier 1981), de quelles combinaisons sont susceptibles des "fils", des "mères ", des "pères » sans équivalents dans les sociétés étudiées ainsi que des « frères » et " sœurs » imaginaires?

La bonne méthode me semble être de ne retenir le vocabulaire de la consanguinité que dans une perspective comparative explicite, et de considérer en concurrence tout ce qui socialement, économiquement ou politiquement constitue le substrat possible du rapport parental en question. L'analyse componentielle, qui peut aider à faire apparaître certains traits de la parenté, doit donc s'accompagner d'une autre démarche qui, écartant le présupposé consanguin, s'efforce de procéder à une analyse sémantique à partir de la recherche des racines des termes vernaculaires de référence et d'adresse et de leur usage éventuel hors du champ parental. Ainsi peuvent apparaître des fonctions sous-tendant des rapports sociaux, indépendantes de la grille génétique ${ }^{20}$.

La traduction des rapports d'affinité, établis par le truchement du lien matrimonial, s'opère selon des approximations analogues, également pleines d'embûches, comme le montrent les travaux d'Émile Benveniste.

En l'état actuel, il me semble donc que malgré la masse énorme de travail accompli, l'étude de la parenté, toujours menée à partir d'analogies terminologiques, reste l'un des champs les plus imprégnés d'ethnocentrisme et, paradoxalement, l'un des plus mal débroussaillés de l'ethnologie ${ }^{21}$.

MOTS CLÉS/KEY WORDS: consanguinité/consanguinity - homologie/homology - généalogie/ genealogy - parentélkinship - soninké.

20. Par exemple, les termes inuit traduits par "cousin" ou "beau-frère" pourraient se rapporter à la notion de collaboration, ou de corésidence, sans relation stricte avec la parenté ou l'affinité (Meillassoux 1993, 1994).

21. Il faut donc accorder une importance particulière à la tentative de Christian Geffray (1990) pour décrire une société domestique, dite matrilinéaire (Makhuwa du Mozambique), sans recourir à la notion de consanguinité, ni dans son principe ni par son vocabulaire, et qui met au jour, par cette méthode, les structures parentales dans leur essence. 
Augé, Marc, ed.

1975 Les domaines de la parenté. Paris, François Maspero («Dossiers africains»).

Benveniste, Émile

1966 Problèmes de linguistique générale.

Paris, Gallimard, 2 vol.

1969 Le vocabulaire des institutions indoeuropéennes. Paris, Éditions de Minuit, 2 vol.

\section{Coult, Allan D.}

1967 «Lineage Solidarity, Transformational Analysis and the Meaning of Kinship

Terminologies ", Man, n.s., 2 (1) : 26-47.

\section{Geffray, Christian}

1990 Ni père ni mère: critique de la parenté. Le cas makhuwa. Paris, Le Seuil.

\section{Glowczewski, Barbara \& Charles-Henry} Pradelles de Latour

1987 "La diagonale de la belle-mère ", L'Homme 104 : 27-53.

\section{Goodenough, Ward H.}

1956 "Componential Analysis and the Study of Meaning ", Language 32 : 195-216.

\section{Graburn, Nelson H. H.}

1964 Taqamiut Eskimo Kinship Terminology. Northern Coordination and Research Center, Department of Northern Affairs and National Ressources, Ottawa, Ontario, Canada.

\section{Héritier, Françoise}

1981 L'exercice de la parenté. Paris, Hautes Études, Gallimard-Le Seuil.

\section{Hirschfeld, Lawrence A.}

1986 « Kinship and Cognition: Genealogy and the Meaning of Kinship Terms ", Current Anthropology 27 (3) : 217-242.

\section{Lounsbury, Floyd G.}

1956 "A Semantic Analysis of the Pawnee Kinship Usage ", Language 32 : 158-194.
1964 "The Formal Analysis of Crow and Omaha-Type Kinship Terminologies ", in Ward H. Goodenough, ed., Explorations in Cultural Anthropology. Essays in the Honor of G. P. Murdock. New York, McGraw-Hill : 351-394.

\section{Meillassoux, Claude}

1993 "Comment se sont perpétués les Inuit ", inPopulation, reproduction, sociétés : perspectives et enjeux de la démographie sociale. Mélanges en l'honneur de Joël Gregory. Montréal, Presses de l'Université de Montréal : 19-47.

1994 «La parenté inuit est-elle sensible à la conception matérialiste de l'histoire?",

L'Ethnographie XC (1), 115 : 115-145.

\section{McLennan, John Ferguson}

1865 Primitive Marriage: An Inquiry into the Origin of the Form of Capture in Marriage Ceremonies. Edinburgh. Réed.

Chicago, University of Chicago Press, 1970.

\section{Morgan, Lewis Henry}

1963 Ancient Society, with an Introduction and Annotations by E. B. Leacock.

Cleveland, The World Publishing Cy. (1st ed. 1877.)

\section{Murdock, George Peter}

1949 Social Structure. New York, The Free Press.

\section{Panoff, Michel}

1965 «La terminologie de la parenté en Polynésie. Essai d'analyse formelle", L'Homme V (3-4) : 60-87.

1976 "Patrifiliation and Practice in a Matrilineal Society ", Ethnology 15 (2) : 175-188.

\section{Rivers, William H.}

1924 Social Organisation. New York, Alfred A. Knopf.

Royal Anthropological Institute of Great Britain and Ireland, ed. 
1874-1964 Notes and Queries on

Anthropology. London, Routledge and

164
Schneider, David M.

1984 A Critique of the Study of Kinship. Ann Arbor, The University of Michigan Press.

\section{Spencer, B.}

1914 Native Tribes of the Northern Territories. London, Macmillan.
Tax, Sol

1937 «Some Problems of Social Organization ", in Fred Eggan, ed., Social Anthropology of the North American Tribes. Chicago, University of Chicago Press.

Wallace, F. C. \& J. A. Atkins

1960 "The Meaning of Kinship Terms ", American Anthropologist 62 (1) : 58-80.

RÉSUMÉ/ABSTRACT

Claude Meillassoux, Parler parenté. — L'usage des termes de parenté existant dans notre vocabulaire pour traduire ceux apparemment homologues des sociétés qu'étudie l'ethnologue, revient à appliquer abusivement à ces sociétés la thèse implicite qui en gouverne notre conceptualisation: la consanguinité. Cette pratique très générale en ethnologie, qu'elle soit fonctionnaliste ou structuraliste, non seulement ne rend pas compte du sens parfois beaucoup plus riche des mots traduits, mais elle dénature et appauvrit le contenu social des faits observés. Même l'analyse componentielle n'échappe pas à cette critique.
Claude Meillassoux, Talking about Kinship. Using our vocabulary's kinship terms to translate the apparently homologous terms of the societies studied by ethnologists amounts to abusively applying to these societies the unspoken thesis underlyning our own conceptualization, namely consanguinity. Besides not adequately explaining the meaning of the translated terms, which may be much more fertile, this widespread practice in ethnology, whether functionalist or structuralist, also alters and impoverishes the social contents of the observed facts. Even componential analyses fall under this criticism. 